\title{
Elderly-Onset Neuromyelitis Optica Spectrum Disorder with Pre-Existing Prednisone Allergy
}

\author{
Christopher Hollen Omer Suhaib Aaron Farrow Evgeny Sidorov \\ Department of Neurology, University of Oklahoma, Oklahoma City, OK, USA
}

\section{Keywords}

Neuromyelitis optica · Systemic corticosteroid allergy · Elderly-onset neuromyelitis optica ·

Optic neuritis

\begin{abstract}
We present a case of an 82-year-old man with new-onset neuromyelitis optica (NMO) spectrum disorder, the treatment of which was complicated by a severe pre-existing prednisone allergy. His age caused much initial doubt about his diagnosis, and his corticosteroid allergy altered our management as we attempted to minimize risk to the patient. Our patient was a healthy 82-year-old, right-handed man who presented with sensory loss of the bilateral lower extremities and progressive, painless vision loss. MRI showed bilateral pre-chiasmatic optic nerve and optic chiasm enhancement, along with enhancement within the thoracic spinal cord from T3 to T7. Serum NMO-IgG was positive with a titer $>1: 100,000$. Due to concern of allergic reaction, our patient initially refused high-dose Solu-Medrol and opted to try plasma exchange alone, but due to worsening of his symptoms we attempted to use dexamethasone as it had a theoretically lower risk of adverse reaction with a known prednisone allergy. There are several cases of elderly-onset NMO in the literature but this is the only case we could find of NMO accompanied by a rare severe allergy to prednisone. This case demonstrates the relative safety of dexamethasone as an alternative to methylprednisolone for acute management of NMO spectrum disorder, though efficacy has not been established in major trials. Cross-reactivity between various systemic corticosteroids is not as well established as topical corticosteroids, so it is difficult to assess the probability of a reaction between prednisone and methylprednisolone.




\section{Case Reports in Neurology}

\begin{tabular}{l|l}
\hline Case Rep Neurol 2018;10:25-28 \\
\hline DOI: $10.1159 / 000485120$ & $\begin{array}{l}\text { C 2018 The Author(s). Published by S. Karger AG, Basel } \\
\text { www.karger.com/crn }\end{array}$ \\
\hline
\end{tabular}

Hollen et al.: Elderly-Onset Neuromyelitis Optica Spectrum Disorder with Pre-Existing Prednisone Allergy

\section{Background}

Neuromyelitis optica (NMO) spectrum disorder is an autoimmune disease of the central nervous system, characterized by inflammation of the optic nerves and longitudinal lesions of the spinal cord. Aquaporin-4-immunoglobulin is highly specific for NMO. The median onset is typically around 40 years of age, though there are several cases in the literature remarkable for onset in patients up to their late 80s [1]. High-dose corticosteroids (methylprednisolone 1,000 mg daily for 3-5 days) are the mainstay of management of initial and acute attacks. Plasma exchange (PLEX) is typically utilized for patients with severe symptoms or those who are not responsive to the initial regimen of corticosteroids. Allergies to systemic corticosteroids are very uncommon, though severe reactions including angioedema and anaphylaxis following oral or intravenous corticosteroids have been described [2].

\section{Case Presentation}

An 82-year-old, right-handed Caucasian man was admitted to our neurology service due to rapidly progressive, painless vision loss and sensory loss of the bilateral lower extremities. Prior to the onset of symptoms he was healthy and independent in his activities of daily living. Initially, his vision began to deteriorate in the left eye 2 weeks prior to presentation, and 1 week later he began to have painless vision loss in the right eye. Subsequently, he began to experience abdominal pain in a band around his umbilicus along with numbness that began in the mid-trunk and gradually extended to include his lower extremities, which ultimately led to his presentation to our emergency department.

His initial examination showed preserved light perception in the left eye with impaired perception of motion and no light perception in the right eye. He had patchy sensory loss to all modalities below T10. He was hyperreflexic in the left lower extremity with crossed adductors, crossed patellar reflex, and an extensor plantar response on the left. He had no discernable weakness or cranial nerve abnormalities. MRI brain/orbits, C-spine, and T-spine with/without contrast revealed bilateral pre-chiasmatic optic nerve and optic chiasm enhancement, along with patchy but longitudinally extensive hyperintense STIR signal and post-contrast enhancement within the thoracic spinal cord from T3 to T7.

Initial laboratory testing was unremarkable, including $\mathrm{CBC}$, comprehensive metabolic panel, $\mathrm{B}_{12}$, copper, rapid plasma regain, antinuclear antibodies, erythrocyte sedimentation rate, and C-reactive peptide. His CSF revealed 10 WBCs, 9 RBCs, glucose 52 (roughly 2/3 serum glucose), and protein 59. CSF oligoclonal bands were negative. Serum angiotensinconverting enzyme was low at 3.

Based on his imaging and CSF findings his working diagnosis was NMO, but neurosarcoidosis was also on our differential given his abnormal age for presentation of NMO. He initially refused steroids, opting for PLEX alone due to the severe reaction he had to oral prednisone 2 months prior (angioedema with airway compromise). He completed 5 rounds of PLEX (every $48 \mathrm{~h}$ for 10 days). However, by hospital day 4 his symptoms worsened. His sensory level now extended from T4 and he began to have weakness of the bilateral lower extremities. Ultimately, his serum NMO-IgG returned positive with a titer $>1: 100,000$, confirming our diagnosis. He reconsidered his position on attempting intravenous steroids given the worsening strength of his upper extremities. Based on the suspicion of lowest probability of cross-reactivity, we initiated dexamethasone, opting for $25 \mathrm{mg}$ every $6 \mathrm{~h}$ followed by 


\section{Case Reports in Neurology}

Hollen et al.: Elderly-Onset Neuromyelitis Optica Spectrum Disorder with Pre-Existing Prednisone Allergy

a taper for 2 days. We moved the patient to an intensive care bed for close monitoring in the event of a severe reaction requiring intubation. His symptoms persisted but did not worsen. However, he suffered from psychosis and hallucinations likely as a consequence of steroid administration on his final 2 days of high-dose dexamethasone. He was ultimately discharged to a rehab facility prior to returning home and arrangements were made to prepare for outpatient rituximab infusions for NMO maintenance therapy.

\section{Discussion}

We present a case of an elderly man with new onset of NMO spectrum disorder. There have been at least 4 cases of elderly-onset NMO with onset after the age of 80 described in the literature, with the oldest at age 90 , but this is the only case we could find of NMO accompanied by a rare severe allergy to prednisone. NMO is characterized by longitudinal lesions of the spinal cord and optic nerves. It follows a relapsing course in the vast majority of patients and is associated with NMO-IgG antibodies, which are highly specific. The 2015 diagnostic criteria for NMO spectrum disorder includes at least 1 core clinical characteristic, a positive test for aquaporin-4-IgG, and exclusion of alternative diagnoses [3]. As has been reported in other cases of NMO in the elderly, the diagnosis was initially placed into some doubt by the patient's age. However, our patient's classic longitudinally extensive thoracic spine lesion, involvement of the bilateral optic nerves/optic chiasm, and lack of history of malignancy provided a high degree of clinical suspicion. Transverse myelitis appears to be the most common initial presenting feature of NMO in the elderly, which is also consistent with late-onset multiple sclerosis where spinal lesions are much more common as compared to early-onset multiple sclerosis [4].

Management of acute attacks of NMO is centered on intravenous corticosteroids. The Neuromyelitis Optica Study Group suggests 5 consecutive days of methylprednisolone followed by an oral steroid taper depending on the severity of the attack and confirmation of NMO diagnosis. If symptoms do not improve, 5-7 cycles of therapeutic PLEX may be beneficial. A study of 21 patients carried out by Menon et al. [5] suggests that intravenous dexamethasone is a fairly comparable, effective, and safe treatment option for patients with demyelinating optic neuritis. However, evidence for the best choice of treatment for acute attacks of NMO is limited.

Corticosteroid allergies are rare, particularly to systemic corticosteroids. Urticarial and dermatologic reactions are most frequent, but anaphylaxis and angioedema can occur in response to corticosteroids [6]. There are four classes of corticosteroids outlined in the Coopman classification of corticosteroids, based on structural composition and dermatologic testing. The Coopman classification system separates methylprednisolone and prednisone in a separate class from dexamethasone, and some small series have suggested hydrocortisone/methylprednisolone may exhibit more cross-reactivity than halogenated steroids like betamethasone or dexamethasone. However, this classification system was designed to address dermatologic reactions to topical steroids and may not adequately reflect potential cross-reactivity between various systemic corticosteroids. Cross-reactivity between systemic corticosteroids has not been well established, in part because the process of creating water-soluble steroids for intravenous use requires coupling to an ester which may modify the allergenicity of corticosteroids [7]. Since we cannot base our clinical counseling on chemical structure or classification to reliably predict potential cross-reactivity, careful challenge testing in a controlled environment must be employed when intradermal testing is not avail- 


\section{Case Reports in Neurology}

able or impractical. Intradermal testing is available to confirm hypersensitivity reactions if suspected, though a graded challenge is not standardized.

In summary, we present a case of an elderly man with NMO with a conundrum of acute management with a previous severe reaction to prednisone. After a review of the available literature, it appeared optimal to initiate intravenous dexamethasone rather than methylprednisolone. Though cross-reactivity between different systemic corticosteroids is not entirely clear, it was felt to be less likely between these two agents. Importantly, in the elderly it is critical to keep NMO in mind in cases of painless vision loss or evidence of typical spinal lesions, even though it is uncommon in this population. Our patient appeared to have no worsening of his symptoms, but steroids were not administered until 14 days after the onset of his vision loss, so it is likely that he did not receive the maximal benefit of acute therapy.

\section{Statement of Ethics}

The authors have no ethical conflicts to disclose.

\section{Disclosure Statement}

The authors have no conflicts of interest to declare. No funding was received for this study.

\section{References}

1 Loh KP, Brennan MJ: Elderly-onset neuromyelitis optica spectrum disorders. J Am Geriatr Soc 2015;63:411-412.

2 Rachid R, et al: Hypersensitivity to systemic corticosteroids: an infrequent but potentially lifethreatening condition. J Allergy Clin Immunol 2011;127:524-528.

-3 Wingerchuk DM, et al: International consensus diagnostic criteria for neuromyelitis optica spectrum disorders. Neurology 2015;85:177-189.

4 Krumbholz M, et al: Very late-onset neuromyelitis optica spectrum disorder beyond the age of 75. J Neurol 2015;262:1379-1384.

5 Menon V, et al: Comparative evaluation of megadose methylprednisolone with dexamethasone for treatment of primary typical optic neuritis. Indian J Ophthalmol 2007;55:355-359.

-6 Erdmann SM, et al: Anaphylaxis induced by glucocorticoids. J Am Board Fam Pract 2005;18:143-146.

7 Torres MJ, Canto G: Hypersensitivity reactions to corticosteroids. Curr Opin Allergy Clin Immunol 2010;10:273-279. 